\title{
The Effect of Maternal Ethanol Ingestion on Fetal Vitamin A in the Rat
}

\author{
MARY A. GRUMMER AND RICHARD D. ZACHMAN \\ University of Wisconsin Perinatal Center, Department of Pediatrics and Nutritional Sciences, Meriter Hospital, \\ Madison, Wisconsin 53715
}

\begin{abstract}
The effect of maternal ethanol ingestion on fetal tissue vitamin A was investigated. Pregnant rats were pair-fed control diets or diets containing $36 \%$ of energy as ethanol. After 17 or 21 d gestation, fetuses were removed and fetal and maternal tissues were analyzed by HPLC for retinol and retinyl palmitate. Ethanol consumption resulted in fewer fetuses per pregnancy, increased number of resorptions, and increased numbers of gross fetal abnormalities. In maternal tissues, ethanol consumption resulted in greater lung and kidney vitamin A concentrations. In the fetuses of ethanol-consuming pregnancies, free retinol in liver was higher at d $\mathbf{1 7}$. However, fetal liver palmitate levels and total retinyl palmitate in liver, lung, and kidney were lower in ethanol-fed rats at d 21 of gestation. Fetal lung retinyl palmitate concentrations were greater at both d 17 and d 21, and kidney levels were also greater at $\mathbf{d} 21$. In conclusion, the ingestion of ethanol by pregnant rats is associated with a reduction in fetal liver vitamin A levels and an elevation in the levels of lung and kidney vitamin $\mathrm{A}$, indicating possible altered vitamin A metabolism as a result of ethanol consumption. (Pediatr Res 28: 186-189, 1990)
\end{abstract}

\section{Abbreviations}

FAS, fetal alcohol syndrome

Ethanol consumption during pregnancy results in an increased incidence of spontaneous abortions (1), an increase in perinatal mortality (2), and a number of congenital malformations (3). The major features of these congenital malformations (FAS) are intrauterine and postnatal growth retardation, craniofacial dysmorphology, congenital heart defects, skeletal anomalies, and CNS dysfunctions (4).

Controversy in the literature still exists over the exact mechanism by which ethanol causes FAS. Some believe ethanol and/ or acetaldehyde have a direct teratogenic effect. The other view is that the abnormalities observed are the result of altered maternal or placental physiology or other nutritional deficiencies caused by the ethanol $(5,6)$.

The interaction of ethanol with vitamin $A$ is a complex interaction. It is known that in both man and animals, chronic consumption of ethanol results in very low levels of liver vitamin A reserves. This can occur despite adequate intake and normal plasma levels of this vitamin $(7-10)$. The pattern of fetal anomalies associated with vitamin A deficiency is similar in some

Received January 29, 1990; accepted April 18, 1990.

Correspondence: Richard D. Zachman, Ph.D, M.D., University of Wisconsin Perinatal Center, Department of Pediatrics and Nutritional Sciences, Meriter Hospital, 202 South Park Street, Madison, WI 53715.

Supported in part by a grant from the Wisconsin Perinatal Foundation and the Cooperative State Research Service, USDA, under agreement 88-37200-3477. respects to that of FAS (11), including frequently observed defects of growth, craniofacial, neurologic, urogenital, and heart systems. Therefore, it is conceivable that vitamin A deficiency could be present in FAS.

The purpose of our research was to determine if maternal ethanol ingestion had an effect on fetal tissue vitamin $A$ in the rat. We found that fetal vitamin A was significantly decreased in liver, but elevated in lung and kidney. Total vitamin A stores were lower in fetuses born of pregnant dams ingesting ethanol for the $21 \mathrm{~d}$ of gestation.

\section{MATERIALS AND METHODS}

Alcohol diet and experimental animals. Female timed-pregnancy Sprague-Dawley rats (Harlan Industries, Indianapolis, IN) were weighed, randomly assigned to groups on d 1 of pregnancy, and kept in individual mesh-bottom cages in a controlled temperature environment with a light-dark cycle of $12 \mathrm{~h}$ light, $12 \mathrm{~h}$ dark. The rats were fed an isocaloric liquid Lieber-DeCarli diet (Dyets, Inc., Bethlehem, PA) containing $25 \%$ protein, $63 \%$ carbohydrate, and $12 \%$ fat; in the treatment diet, ethanol isocalorically replaced carbohydrate. Diets contained $6 \mathrm{IU} / \mathrm{kg}$ diet of retinyl acetate. Ethanol-fed rats were started on a diet containing $12 \%$ ethanol on $\mathrm{d} 1$, then $24 \%$ ethanol on $\mathrm{d} 2-3$, then $36 \%$ ethanol for the remaining days of the experiment. Rats on the control diet were pair-fed, receiving the average amount of food consumed by the ethanol-fed rats the previous day. The diet was changed daily and fed approximately at the beginning of the dark cycle. Food intake was recorded daily and body wt, 1-2 times per wk $(9,10)$.

After either 17 or $21 \mathrm{~d}$ gestation, rats from both the control and ethanol groups were anesthetized with ether, and blood was obtained by heart puncture for ethanol determinations. The fetuses were then removed from the uterus, decapitated, and weighed. Any anomalies and resorbed fetuses were noted at this time. The mother rats were then killed by heart/lung removal, and then other maternal and fetal tissues were immediately removed, rinsed, blotted dry, and frozen. All tissues were stored protected from light at $-20^{\circ} \mathrm{C}$ for no longer than 2 mo until analysis. Blood ethanol levels were measured by the oxidation of ethanol by alcohol dehydrogenase with simultaneous reduction of NAD (12).

Tissue extraction and vitamin $A$ analysis. Weighed portions of tissues were homogenized and extracted with standard chloroform methanol procedures (13). Portions of the extract containing $0.25 \mathrm{mg} / \mathrm{mL}$ butylated hydroxytoluene were assayed by use of HPLC. Retinol and retinyl palmitate were easily separated with a $80 \%$ methanol, $15 \% \mathrm{CHCl}_{3}$, and $5 \%$ water mobile phase. Retinyl palmitate and retinyl oleate are not separated in this system, and therefore were calculated together. Amounts of retinol and retinyl palmitate are quantitated from HPLC peak areas determined from standard curves. Repeated addition of retinyl acetate as an internal standard to homogenates before 
extraction indicated that the extraction procedure provided essentially $100 \%$ recovery.

Two separate groups of pregnant rats were used in the data reported here. The fetal tissues from each mother were pooled for d 17 rats. For d 21, the fetal tissues from the 1st group of mothers were analyzed individually; the fetal tissues from the 2 nd group of mothers were pooled. Data were analyzed by analysis of variance using a general linear model with the computerized Statistical Analysis System program (SAS Institute, Inc., Cary, NC) (14). The randomized complete block design designated the two separate groups of rats as blocks, and in the case of $\mathrm{d} 21$ fetal data, a nested classification was used because the fetuses from one group were analyzed individually. Comparisons were made between control and ethanol groups and not between $\mathrm{d} 17$ and 21 data.

\section{RESULTS}

Maternal ethanol consumption effects on pregnancy. Ethanol consumption by pregnant rats resulted in markedly elevated blood ethanol levels of $58 \pm 9 \mathrm{mmol} / \mathrm{L}$. No ethanol $(<4 \mathrm{mmol} /$ $\mathrm{L}$, sensitivity of method) was detectable in controls. Ethanol consumption also resulted in fewer fetuses per pregnancy, an increased number of resorptions, and increased numbers of obvious gross fetal deformities (Table 1). There was no difference in total food intake and no difference in final maternal wt due to ethanol consumption, although wt gain/d was lower in ethanol-fed rats. Fetal wt was greater at d 17 and lower at d 21 in ethanol groups.

Effect of Maternal Ethanol Consumption on Tissue Vitamin A Levels. Maternal tissues. Liver retinyl palmitate was insignificantly $(p=0.06)$ lower in maternal rats ingesting ethanol at $\mathrm{d}$ 21 gestation (Table 2). In lung and kidney, both retinol and retinyl palmitate concentrations were greater in ethanol-ingesting mothers at $\mathrm{d} 17$ and 21 gestation, and most increases were also apparent at $17 \mathrm{~d}$ gestation. Total maternal storage of retinyl palmitate was lower at $d 21$ gestation.

Fetal tissues. Differences in fetal tissue vitamin A were evident (Table 3). Liver retinol concentrations were greater at $d 17$ in ethanol-fed animals than in controls. Retinol levels increased in both groups but were not significantly different at d 21 . Liver retinyl palmitate levels were similar at $\mathrm{d} 17$, and increased from d 17 to 21 in control fetuses. No increase in retinyl palmitate occurred in ethanol-fed rats from d 17 to 21 .

In lung, the concentration of retinyl palmitate decreased from $\mathrm{d} 17$ to 21 in control fetuses. However, in lungs of fetuses from ethanol-consuming mothers, the retinyl palmitate levels were greater than controls at $\mathrm{d} 17$ and 21 , with no decrease from $\mathrm{d} 17$ to 21 . The kidney was similar to lung; retinol and retinyl pal- mitate levels were elevated in ethanol-fed rats at $d 21$. Day 17 kidneys were too small to analyze.

The total retinol per fetus was greater in ethanol-fed rats at $d$ 17. By d 21, the total storage per fetus of retinyl palmitate was $50 \%$ lower in fetuses of ethanol-consuming rats compared with controls.

\section{DISCUSSION}

The economic cost of FAS in the United States alone is estimated at several million dollars annually, and may account for over $10 \%$ of the residential care costs of institutionalized mentally retarded patients (15). The actual mechanism of FAS is unknown. There is still controversy over whether it is the result of direct ethanol teratogenesis, or if it occurs by an indirect mechanism resulting from specific nutritional deficiencies.

There are no published data on the effects of maternal ethanol consumption on fetal vitamin A content. This is the first report on vitamin A levels in fetuses and female rats ingesting ethanol during pregnancy. Chronic ethanol consumption in adult humans and adult animals results in overall vitamin A deficiency and redistribution of the vitamin in various organs (10,16-18). Because FAS and both vitamin A deficiency (11) and excess (19) have some similar organ teratogenic and growth defects, we set out to study the effect of maternal ethanol ingestion on fetal vitamin A stores.

The method used here for adult rat ethanol ingestion is well established $(7,9,10,20)$. Although diets and methods of feeding commonly used in rodent studies are devised to control for adequate and equivalent calorie, protein, and other nutrient intake, it has been shown that ethanol-fed rats may not gain as much weight as controls (21). The mechanism for this occurrence is not clear, but it does not appear that this difference in weight gain accounts for the effects of ethanol.

The ingestion of ethanol resulted in a decrease, although not statistically significant, in maternal liver vitamin A stores. This trend reflects the larger decreases observed in male adult rats fed $30-36 \%$ ethanol for $4-6$ wk $(7,9,10,17,18)$. The redistribution and increased vitamin $A$ in adult pregnant female rat lung and kidney with ethanol consumption is similar to that found previously in male rats $(7,10,18)$. The mechanism for increased extrahepatic vitamin A accumulation has not been established.

Maternal ethanol ingestion was associated with a significant depletion of fetal tissue vitamin A compared with fetal tissue of control pregnancies. Therefore, the fetus did not respond to ethanol ingestion as a maternal extrahepatic organ that accumulates vitamin A with ethanol ingestion $(7,10)$. Instead, there was a large deficit of fetal liver stores, rather than the expected increase found in normal gestation $(22,23)$. There was also some redistribution of the vitamin, resulting in elevated concentrations

Table 1. General effects of maternal ethanol consumption on pregnancy*

\begin{tabular}{|c|c|c|c|c|}
\hline & \multicolumn{2}{|c|}{ d 17} & \multicolumn{2}{|c|}{ d 21} \\
\hline & Control & Ethanol & Control & Ethanol \\
\hline Number of rats & 6 & 5 & 5 & 7 \\
\hline Live fetuses/rat & $11.0 \pm 1.5$ & $7.4 \pm 1.9$ & $10.6 \pm 0.8$ & $6.4 \pm 1.8^{a}$ \\
\hline Total live fetuses & 66 & 37 & 53 & 45 \\
\hline Fetuses resorbed/rat & $0.2 \pm 0.2$ & $1.8 \pm 0.5^{a}$ & $0.2 \pm 0.2$ & $2.0 \pm 0.5^{a}$ \\
\hline Obvious anomalies & 0 & $1 \uparrow$ & 0 & $3 \dagger$ \\
\hline Fetal wt $(\mathrm{g})$ & $0.70 \pm 0.07$ & $0.86 \pm 0.06^{b}$ & $4.65 \pm 0.18$ & $3.58 \pm 0.16^{b}$ \\
\hline Final maternal wt (g) & $246.0 \pm 7.4$ & $236.6 \pm 6.1$ & $280.4 \pm 14.6$ & $253.3 \pm 13.6$ \\
\hline Maternal gain/d (g) & $4.2 \pm 0.4$ & $3.3 \pm 0.2^{a}$ & $5.2 \pm 0.5$ & $3.4 \pm 0.5^{a}$ \\
\hline Feed intake $(\mathrm{g})$ & $929.0 \pm 9.3$ & $937.2 \pm 14.6$ & $1182.6 \pm 16.6$ & $1211.3 \pm 44.4$ \\
\hline Blood ethanol levels (mmol/L) & nd & nd & $\ddagger$ & $58 \pm 9$ \\
\hline
\end{tabular}

* Values represent means \pm SEM. Treatment means for $\mathrm{d} 17$ or for $\mathrm{d} 21$ not sharing a common superscript letter differ significantly: ${ }^{a}<0.05$,

" $<0.005$. nd, not determined.

$\dagger$ Anomalies observed included gastroschisis, skeletal abnormalities, hydrops fetalis, skull hemorrhage, and retarded growth.

$\ddagger$ Ethanol undetectable below $4 \mathrm{mmol} / \mathrm{L}$. 
Table 2. Effect of maternal ethanol consumption on maternal tissue vitamin A levels (nmol/g wet wt $\pm S E M)^{*}$

\begin{tabular}{|c|c|c|c|c|}
\hline & \multicolumn{2}{|c|}{ d 17} & \multicolumn{2}{|c|}{ d 21} \\
\hline & Control & Ethanol & Control & Ethanol \\
\hline \multicolumn{5}{|l|}{ Liver } \\
\hline Retinol & $71.15 \pm 11.57$ & $75.14 \pm 9.79$ & $85.35 \pm 9.02$ & $118.46 \pm 21.61$ \\
\hline Retinyl palmitate & $1066.92 \pm 58.43$ & $1080.24 \pm 73.01$ & $1105.42 \pm 117.94$ & $766.89 \pm 62.52$ \\
\hline \multicolumn{5}{|l|}{ Lung } \\
\hline Retinol & $3.43 \pm 0.49$ & $7.20 \pm 0.91^{c}$ & $2.87 \pm 0.38$ & $7.62 \pm 0.80^{c}$ \\
\hline Retinyl palmitate & $11.19 \pm 1.64$ & $23.74 \pm 2.62^{c}$ & $8.50 \pm 0.87$ & $22.24 \pm 3.22^{c}$ \\
\hline \multicolumn{5}{|l|}{ Kidney } \\
\hline Retinol & $3.29 \pm 0.24$ & $5.14 \pm 0.31^{b}$ & $2.24 \pm 0.24$ & $6.54 \pm 0.56^{c}$ \\
\hline Retinyl palmitate & $2.27 \pm 0.56$ & $2.03 \pm 0.24$ & $0.77 \pm 0.03$ & $2.41 \pm 0.14^{c}$ \\
\hline \multicolumn{5}{|l|}{ Totals $(\mu \mathrm{mol})$} \\
\hline Retinol & $0.72 \pm 0.13$ & $0.69 \pm 0.09$ & $0.84 \pm 0.11$ & $1.33 \pm 0.35$ \\
\hline Retinyl palmitate & $10.53 \pm 0.48$ & $9.67 \pm 0.69$ & $10.54 \pm 0.86$ & $7.92 \pm 0.30^{a}$ \\
\hline
\end{tabular}

${ }^{*}$ Treatment means for $\mathrm{d} 17$ or for $\mathrm{d} 21$ not sharing a common superscript letter differ significantly: ${ }^{a}<0.05,{ }^{b}<0.01,{ }^{c}<0.001$.

Table 3. Effect of maternal ethanol consumption on fetal tissue vitamin A levels $(\mathrm{nmol} / \mathrm{g} \text { wet } w t \pm S E M)^{*}$

\begin{tabular}{|c|c|c|c|c|}
\hline & \multicolumn{2}{|c|}{ d 17} & \multicolumn{2}{|c|}{ d 21} \\
\hline & Control & Ethanol & Control & Ethanol \\
\hline \multicolumn{5}{|l|}{ Liver } \\
\hline Retinol & $2.87 \pm 0.14$ & $3.78 \pm 0.17^{a}$ & $9.90 \pm 1.19$ & $10.73 \pm 1.12$ \\
\hline Retinyl palmitate & $28.01 \pm 3.11$ & $28.25 \pm 2.45$ & $43.43 \pm 2.10$ & $28.53 \pm 2.38^{a}$ \\
\hline \multicolumn{5}{|l|}{ Lung } \\
\hline Retinol & $1.36 \pm 0.17$ & $1.78 \pm 0.28$ & $1.68 \pm 0.03$ & $3.43 \pm 0.25^{b}$ \\
\hline Retinyl palmitate & $9.37 \pm 1.05$ & $14.72 \pm 1.71^{c}$ & $5.07 \pm 0.31$ & $15.84 \pm 1.54^{b}$ \\
\hline \multicolumn{5}{|l|}{ Kidney } \\
\hline Retinol & nd & nd & $4.65 \pm 0.77$ & $8.46 \pm 0.73^{a}$ \\
\hline Retinyl palmitate & & & $5.24 \pm 0.35$ & $9.55 \pm 0.98^{a}$ \\
\hline \multicolumn{5}{|l|}{ Total (nmol/fetus) $\dagger$} \\
\hline Retinol & $0.15 \pm 0.02$ & $0.22 \pm 0.03^{c}$ & $2.81 \pm 0.37$ & $1.99 \pm 0.20$ \\
\hline Retinyl palmitate & $1.51 \pm 0.31$ & $1.64 \pm 0.23$ & $11.40 \pm 0.41$ & $5.81 \pm 0.39^{b}$ \\
\hline
\end{tabular}

* Treatment means for $\mathrm{d} 17$ or for d 21 not sharing a common superscript letter differ significantly: ${ }^{a}<0.005,{ }^{b}<0.001,{ }^{c}<0.01$. nd, Retinol and retinyl palmitate not determined in $\mathrm{d} 17$ kidneys.

$\dagger$ Includes liver and lung only at d 17; liver, lung, and kidney at $\mathrm{d} 21$ gestation.

in lung and kidney. The lower total fetal vitamin A level with maternal ethanol ingestion is not likely to be a mere reflection of a vitamin A deficit in the mother in these experiments. First, caloric and vitamin A intake was nearly equal (Table 1), and second, the supply of vitamin A to the fetus has been shown to be somewhat regulated. This is quite insensitive to variations in maternal vitamin A stores, except at levels far below the storage levels in our study $(24,25)$.

Differences resulting from ethanol ingestion that were evident at d 17 and differences occurring from d 17 to 21 suggest alterations in vitamin A metabolism in fetuses due to maternal ethanol consumption. At d 17 in fetuses of ethanol-consuming mothers, liver retinol concentrations and total retinol per fetus were elevated and lung retinyl palmitate was greater. From d 17 to 21 , the normal increase in liver and decrease in lung retinyl palmitate did not occur $(23,26)$. The marked retinol and retinyl palmitate changes in these fetuses suggest that the normal metabolic pathways for vitamin A have been affected by the maternal ethanol consumption.

The reason for the decreased vitamin A in liver is possibly a stimulation of retinyl ester hydrolase activity by ethanol, causing mobilization of liver retinyl palmitate stores, as suggested by others $(17,27)$. This increase in mobilization could also possibly result in accumulation of vitamin $A$ in extrahepatic tissues. An increase in retinol metabolism due to an induction of hepatic microsomal enzymes by ethanol may also have been involved in hepatic vitamin A depletion (28). Altered placental transport of the vitamin due to potential ethanol damage (29) is another potential explanation for the findings. However, such mechanism issues were not addressed in our experiments.

In conclusion, the ingestion of ethanol by pregnant rats is associated with decreased fetal liver and increased lung and kidney tissue vitamin A levels. A number of these changes, evident by d 17 of gestation, suggest alterations in vitamin A metabolism in fetuses of ethanol-consuming mothers. Additional changes in the metabolism of retinol could be taking place, such as an increased formation of potent teratogenic metabolites, such as retinoic acid (19), that could produce toxic changes at the cellular level. Whether or not differences in organ vitamin A content occur earlier in gestation where organogenesis might be affected is unknown. Studies will have to be extended to answer such questions.

Acknowledgment. The authors thank Rebecca Landhough for her assistance in the statistical analysis. REFERENCES

1. Abel E 1984 Prenatal affects of alcohol. Drug Alcohol Depend 14:1-10

2. Kaminski M, Rumeau C, Schwartz D 1978 Alcohol consumption in pregnant women and the outcome of pregnancy. Alcoholism: Clin Exp Res 2:155163 
3. Jones K, Smith DW 1973 Recognition of the fetal alcohol syndrome in early infancy. Lancet 2:999-100

4. Clarren SK, Smith DW 1978 The fetal alcohol syndrome. N Engl J Med 298:1063-1067

5. Dow KE, Riopelle RJ 1987 Neurotoxicity of ethanol during perinatal development. Clin Neuropharmacol 10:330-341

6. Sokol RJ, Abel EL 1988 Alcohol-related birth defects: outlining current research opportunities. Neurotoxicol Teratol 10:183-186

7. Sato M, Lieber CS 1981 Hepatic vitamin A depletion after chronic ethanol consumption in baboons and rats. J Nutr 11:2015-2023

8. Leo MA, Lieber CS 1983 Interaction of ethanol and vitamin A. Alcoholism: Clin Exp Res 7:15-21

9. Grummer MA, Erdman JW 1983 Effect of chronic alcohol consumption and moderate fat diet on vitamin $A$ status in rats fed either vitamin $A$ or $\beta$ carotene. J Nutr 113:350-364

10. Grummer MA, Erdman JW 1986 Effect of chronic ethanol consumption and moderate or high fat diet upon tissue distribution of vitamin $\mathrm{A}$ in rats fed either vitamin $A$ or $\beta$-carotene. Nutr Res 6:61-73

11. Wilson JG, Roth CB, Warkamy J 1953 An analysis of the syndrome of malformations induced by maternal vitamin A deficiency. Effects of restoration of vitamin A at various times during gestation. Am J Anat 92:189217

12. Poklis A, Markell MA 1982 Evaluation of a modified alcohol dehydrogenase assay for the determination of ethanol in blood. Clin Chem 28:2125-2127

13. Bligh EG, Dyer WJ 1959 A rapid method of total lipid extraction and purification. Can J Biochem Physiol 37:911-917

14. Ray AA 1981 SAS Users Guide: Statistics. SAS Institute, Inc, Cary, NC

15. Abel EL, Sokol RJ 1987 Incidence of fetal alcohol syndrome and economic impact of FAS-related anomalies. Drug Alcohol Depend 19:51-80

16. Lieber CS, Leo MA 1986 Interaction of ethanol with vitamin A deficiency and excess. In: Scarpelli DG, Migaki G (eds) Nutritional Diseases: Research Directions in Comparative Pathobiology. Alan R Liss, Inc, pp 283-318
17. Rasmussen M, Blomhoff R, Helgerud P, Solberg LA, Berg T, Norum KR 1985 Retinol and retinyl esters in parenchymal and nonparenchymal rat liver cell fractions after long term administration of ethanol. J Lipid Res 26:1112 1119

18. Leo MA, Kim C, Lieber CS 1986 Increased vitamin A in esophagus and other extrahepatic tissues after chronic ethanol consumption in the rat. Alcoholism: Clin Exp Res 10:487-492

19. Kochhar DM, Penner JD, Satre MA 1988 Derivation of retinoic acid and metabolites from a teratogenic dose of retinol (vitamin A) in mice. Toxicol Appl Pharmacol 96:429-441

20. Weinberg J 1985 Effects of ethanol and maternal nutritional status on fetal development. Alcoholism: Clin Exp Res 9:49-55

21. Lieber CS, DeCarli LM 1986 The feeding of ethanol in liquid diets. Alcoholism: Clin Exp Res 10:550-553

22. Ismadi SD, Olson JA 1982 Dynamics of the fetal distribution and transfer of vitamin $A$ between rat fetuses and their mother. Int $J$ Vitam Nutr Res 52:111-118

23. Zachman RD, Kakkad B, Chytil F 1984 Perinatal rat lung retinol (vitamin A) and retinyl palmitate. Pediatr Res 18:1297-1299

24. Moore T 1971 Vitamin A transfer from mother to offspring in mice and rats. Int J Vitam Nutr Res 41:301-306

25. Takahashi YI, Smith JE, Winick M, Goodman DS 1975 Vitamin A deficiency and fetal growth and development in the rat. J Nutr 105:1299-1310

26. Shenai JP, Chytil F 1990 Vitamin A storage in lungs during perinatal development in the rat. Biol Neonat 57:126-132

27. Friedman HI, Mobarham S, Hupert J, Lucchixi C, Henderson C, Lanzenberg $\mathrm{P}$, Layden TJ 1989 In vitro stimulation of rat liver retinyl ester hydrolase by ethanol. Arch Biochem Biophys 269:69-74

28. Leo MA, Lieber CS 1985 New pathway for retinol metabolism in liver microsomes. J Biol Chem 260:5228-5231

29. Fisher SE 1988 Selective fetal malnutrition: The fetal alcohol syndrome. J Am Coll Nutr 7:101-106 